\title{
Rejuvenating $\mathrm{T}$ cells
}

\section{By Tracey Baas, Senior Editor}

Two Japanese teams have used induced pluripotent stem cells to regenerate antigen-specific $\mathrm{T}$ cells in patients whose immune systems have been depleted. ${ }^{1,2}$ Megakaryon Corp. is developing and commercializing the technology of one group to produce induced pluripotent stem cellderived human $\mathrm{T}$ cells.

$\mathrm{T}$ cells play an important role in the host response to viral infections and cancer, but chronic exposure to viral or cancer antigens can drive $\mathrm{T}$ cells into an exhausted state in which they lose their effector function and capacity to proliferate.

Multiple groups have tried to expand patients' antigen-specific T cells ex vivo in order to boost levels of antigen-responsive $\mathrm{T}$ cells for adoptive immunotherapy to help patients mount a response to a virus or cancer. ${ }^{3}$ However, these expanded $\mathrm{T}$
"Only immunotherapy will be capable of completely getting rid of metastatic cancers, while conventional methods such as surgery, chemotherapy or radiation cannot."

-Hiroshi Kawamoto, Kyoto University

\section{Getting ready for the clinic}

Both teams now plan to test the safety and efficacy of the regenerated antigen-specific human $\mathrm{T}$ cells in animal models of cancer or infectious diseases.

Kawamoto thinks the method will be best suited for treating metastatic cancers.

"Only immunotherapy will be capable of completely getting rid of metastatic cancers, while conventional methods such as surgery, chemotherapy or radiation cannot," he said.

"I think they will next need to investigate cells have not been effective due to loss of function.

The most successful studies occurred in patients with advanced melanoma treated with $\mathrm{CD}^{+} \mathrm{T}$ cell adoptive immunotherapy, in which eradication of tumors correlated with increased presence of stem celllike $\mathrm{CD}^{+}{ }^{+}$cells. ${ }^{4}$

These results collectively suggest that using induced pluripotent stem (iPS) cell technology to generate large numbers of antigen-specific CD8 ${ }^{+}$ T cells would enhance the effects of adoptive immunotherapy.

Several groups have generated T cell-derived iPS cells, ${ }^{5-8}$ but not much is known about differentiating these cells back into antigen-specific T cells.

In the new studies, two Japanese groups used mature, antigenspecific $\mathrm{CD}^{+} \mathrm{T}$ cells and reprogrammed them into iPS cells (see Figure 1, "Adoptive T cell-based immunotherapy strategies"). These iPS cells showed typical characteristics of pluripotency such as embryonic stem cell (ESC)-like morphology, capacity for teratoma formation, endogenous expression of the transcription factors used in reprogramming and disappearance of the T cell markers CD3 and CD8.

The Tokyo group, led by Hiromitsu Nakauchi, used mature HIV p27 (nef)-specific $\mathrm{CD}^{+} \mathrm{T}$ cells obtained from a patient infected with HIV-1 to produce iPS cells. The other group, led by Hiroshi Kawamoto, used a melanoma patient-derived $\mathrm{T}$ cell line expressing the melanoma epitope melan-A (MLANA; MART1) to produce iPS cells.

Nakauchi is professor and director at the Center for Stem Cell Biology Regenerative Medicine at the Institute of Medical Science at The University of Tokyo. Kawamoto did the research when he was team leader at the laboratory for lymphocyte development at the RIKEN Research Center for Allergy and Immunology. He now is a professor of immunology at the Institute for Frontier Medical Sciences at Kyoto University. the efficiency and the ease of generating antigen-specific $\mathrm{T}$ cells from a patient's blood and establish the safety of the therapy by using in vivo models," said Keiichi Fukuda, professor at the Keio University School of Medicine. His laboratory was one of the first to generate iPS cells from human circulating T cells.?

Because contamination with nonhuman cellular products could potentially induce immunorejection, "for clinical applications, a xenofree iPS cell culture condition is desirable," he noted.

Kawamoto agreed that the mouse feeder cells will need to be removed. "A method to induce human early hematopoietic progenitors from iPS cells by a feeder-free system has already been established by Cellular Dynamics International Inc." he noted. "Our team is also developing a system that induces $\mathrm{T}$ cell progenitors from early hematopoietic progenitors in feeder-free condition based on a method that we reported in Science in 2010. By combining the two ideas, we are now setting up a complete feeder-free and xeno-free system." Kawamoto published a paper on differentiating murine hematopoietic progenitors into T cells. ${ }^{9}$

Cellular Dynamics markets the iCell platform for differentiating iPS cells into homogeneous, functional cell types. The company also markets human iPS cell-derived iCell cardiomyocytes, endothelial cells, neurons and hepatocytes, and has iCell hematopoietic cells in development.

Nakauchi thinks excluding xenogeneic materials may not be an absolute requirement for clinical trials. "We already have GMP-quality mouse feeder cells," he said.

He did say that because of tumorigenic risk from residual undifferentiated cells, "for the first-in-man clinical study, and to ensure safety, 
Figure 1. Adoptive T cell-based immunotherapy strategies. Two teams reprogrammed antigen-specific $\mathrm{CD} 8^{+} \mathrm{T}$ cells (orange cells) using Yamanaka factors and SV40 large T antigen to produce induced pluripotent stem (iPS) cells (purple cells). When cocultured with mouse feeder cells, the iPS cells differentiated into mature, antigen-specific CD8 ${ }^{+}$ T cells (blue cells). These cells could be expanded using the T cell growth factor IL-2, and the result was a source of antigen-specific $T$ cells that showed longer telomeres, greater effector function and higher proliferating capacity than antigen-specific T cells that did not go through the iPS cell stage (orange cells).

Standard adoptive $\mathrm{T}$ cell-based immunotherapy involves expanding CD8 ${ }^{+} \mathrm{T}$ cells ex vivo with IL-2. The cells can be infused back into the patient to help them mount a response to a virus or cancer, but the approach has shown limited efficacy. (Figure based on Figure 1B in ref. 12.)

we are planning to install a suicide-gene system that can be activated by administration of a drug like anciclovir."

Previous studies have shown that by incorporating expression of herpes simplex virus thymidine kinase (HSV-tk) in ESCs, any teratoma formation can be eliminated by dosing with ganciclovir, a drug that targets HSV-tk. ${ }^{10,11}$

Kawamoto agreed that introducing a suicide gene was a good idea and said his group was thinking of doing the same with its system.

\section{Stepping into stem cells}

Megakaryon was founded in September 2011 by a group including iPS Academia Japan Inc. and iCELL Inc., which are providing seed capital and are making their IP available to Megakaryon.

iPS Academia Japan manages patents and other IP related to iPS cell technology developed by Shinya Yamanaka of Kyoto University and colleagues. iCELL was established to manage the IP related to ESC and iPS cell technologies developed by Nakauchi and colleagues.

Yamanaka, who won last year's Nobel Prize in medicine for his discovery that mature cells can be reprogrammed to become pluripotent, serves on Megakaryon's scientific advisory board.

Megakaryon is headquartered in Japan and plans to establish a U.S. presence to support the development and commercialization of its ESCand iPS cell-derived products.

The company's most advanced project relates to iPS cell-derived platelets. Platelets obtained from blood donations cannot be frozen and have a shelf life of only five days after collection. Platelet shortages could be avoided by using human iPS cells to produce large quantities of platelets.

The company plans to start clinical studies within two years.

According to Genjiro Miwa, representative director of Megakaryon, "The pipeline also includes $\mathrm{T}$ cell and red blood cell projects, which are about two years behind the platelet project."

A patent application has been filed by the University of Tokyo for the work of the Nakauchi team. The RIKEN Research Center for Allergy and Immunology has patented the work by Kawamoto's team, and it is available for licensing.

Baas, T. SciBX 6(5); doi:10.1038/scibx.2013.107

Published online Feb. 7, 2013

\section{REFERENCES}

1. Nishimura, T. et al. Cell Stem Cell; published online Jan. 3, 2013; doi:10.1016/j.stem.2012.11.002

Contact: Hiromitsu Nakauchi, The University of Tokyo, Tokyo, Japan e-mail: nakauchi@ims.u-tokyo.ac.jp

2. Vizcardo, R. et al. Cell Stem Cell; published online Jan. 3, 2013; doi:10.1016/j.stem.2012.11.006

Contact: Hiroshi Kawamoto, RIKEN Research Center for Allergy and Immunology, Yokohama, Japan e-mail: kawamoto@rcai.riken.jp

3. June, C.H. J. Clin. Invest. 117, 1466-1476 (2007)

4. Rosenberg, S.A. Nat. Rev. Clin. Oncol. 8, 577-585 (2011)

5. Brown, M.E. et al. PLoS ONE 5, e11373; published online June 29, 2010; doi:10.1371/journal.pone.0011373

6. Loh, Y.-H. et al. Cell Stem Cell 7, 15-19 (2010)

7. Seki, T. et al. Cell Stem Cell 7, 11-14 (2010)

8. Staerk, J. et al. Cell Stem Cell 7, 20-24 (2010)

9. Ikawa, T. et al. Science 329, 93-96 (2010)

10. Rong, Z. et al. J. Biol. Chem. 287, 32338-32345 (2012)

11. Martz, L. SciBX 5(34); doi:10.1038/scibx.2012.890

12. Crompton, J.G. et al. Cell Stem Cell 12, 6-8 (2013)

\section{COMPANIES AND INSTITUTIONS MENTIONED}

Cellular Dynamics International Inc., Madison, Wis.

iCELL Inc., Tokyo, Japan

iPS Academia Japan Inc., Kyoto, Japan

Keio University School of Medicine, Tokyo, Japan

Kyoto University, Kyoto, Japan

Megakaryon Corp., Tokyo, Japan

RIKEN Research Center for Allergy and Immunology,

Yokohama, Japan

The University of Tokyo, Tokyo, Japan 